\title{
Choosing a Mass Immunization Program against Meningococcal B
}

This interactive feature addresses the approach to a clinical issue. A case vignette is followed by specific options, neither of which can be considered either correct or incorrect. In short essays, experts in the field then argue for each of the options. Readers can participate in forming community opinion by choosing one of the options and, if they like, providing their reasons.

\section{CASE VIGNETTE \\ An Outbreak of Meningococcal Disease}

Ken Wu, M.B., B.S.

You are a physician advising the national health agency in your country (comparable to the Centers for Disease Control and Prevention $[C D C]$ in the United States). An outbreak of meningitis has occurred among students in a public university. Four cases of invasive meningococcal disease caused by Neisseria meningitidis serogroup B have been reported to the national health agency within the past month. All the affected patients were undergraduate students between 19 and 21 years of age who were otherwise healthy. They had had all the routine childhood immunizations recommended by the national health agency but were not immunized against meningococcal B. All four patients were hospitalized and treated successfully and had no permanent complications. Reports of the outbreak have made local and national news and are trending on social media, causing a high level of anxiety among parents, especially those with children graduating from high school.

In the United States, there have been 10 university-based outbreaks of meningococcal B disease in seven states since 2013; these outbreaks have involved a total of 39 cases and 2 deaths. ${ }^{1}$ In response, affected universities have initiated meningococcal $\mathrm{B}$ vaccination campaigns, with mass vaccination of university students recommended in 8 of the outbreaks. Since its development, the vaccine against meningococcal $\mathrm{B}$ has become part of the routine immunization schedule in some jurisdictions; for example, it is included in the routine immunization schedule in the United Kingdom for all infants younger than 1 year of age and in the Australian state of South Australia for all infants younger than 1 year of age and for adolescents 16 to 17 years of age. ${ }^{2,3}$ The current policy of the health agency in your country regarding administration of the meningococcal $\mathrm{B}$ vaccine to adolescents and young adults 16 to 23 years of age who are not at increased risk is that the decision about vaccination should be based on individual clinical factors.

Your national health agency is asking for your advice about whether to expand immunization for meningococcal B by recommending administration of the vaccine to all persons 17 to 18 years old in addition to a mass vaccination campaign at universities at which there is an outbreak. In particular, you are asked to consider the effectiveness of the vaccine in preventing both meningococcal B disease and transmission of infection, as well as the cost-effectiveness of a mass vaccination program.

\section{TREATMENT OPTIONS}

Which one of the following choices would you recommend to the national health agency? Base your choice on the published literature, your own experience, published guidelines, and other information sources.

1. Administer the meningococcal $B$ vaccine to all 17-to-18-year-olds and to all students at universities with an outbreak.

2. Administer the meningococcal B vaccine only to students at universities with an outbreak.

To aid in your decision making, each of these approaches is defended in a short essay by an expert in the field. Given your knowledge of the issue and the points made by the experts, which approach would you choose? 
OPTION I

Administer the Meningococcal B Vaccine to All 17-to-18-Year-Olds and to All Students at Universities with an Outbreak

E. Kim Mulholland, M.D.

Meningococcal B disease is a curious and terrifying condition with unusual epidemiology. Sporadic cases occur throughout the world, with occasional small outbreaks, but large-scale epidemics, when they occur, can last for a decade or more. Unlike other meningococcal serogroups, meningococcal B is not amenable to the highly successful vaccine-development approach that is based on covalent binding of the capsular polysaccharide to a carrier protein to form a polysaccharide-protein conjugate vaccine. Vaccines developed in the Americas and in Norway in response to previous meningococcal B epidemics were based on the outer-membrane vesicle approach using vesicles of the bacterial membrane that express important epitopes, particularly the immunodominant PorA protein, which varies from epidemic to epidemic. ${ }^{4}$ The $1990-$ 2005 epidemic in New Zealand was successfully aborted by a tailor-made outer-membrane vesicle vaccine, which was administered to a high proportion of persons younger than 20 years of age in the country. ${ }^{5}$ That vaccine has since been modified to produce one of the two currently available vaccines that cover most strains of meningococcal B - 4CMenB (Bexsero, GlaxoSmithKline). The other meningococcal B vaccine currently in use is MenB-FHbp (Trumenba, Pfizer) which targets factor $\mathrm{H}$ binding protein.

Although meningococcal B disease can affect all ages, the two age groups at highest risk are young infants and older teenagers. The organism circulates easily in the 17-to-18-year-old age group because of the ease with which persons of this age share saliva ${ }^{6}$ - a point that may be well understood by readers who are parents of teenagers or those with fond memories of those years. Sadly, this behavior carries with it a small risk of the horrors of meningococcal B disease, which often leads to serious sequelae, such as multiple limb amputations, or to death. ${ }^{7}$ It is also believed to be the means by which meningococcal B circulates in a community; therefore, prevention of carriage in this age group should lead to herd immunity. Unfortunately, findings of a large study by Marshall et al. published in this issue of the Journal provide convincing evidence that the $4 \mathrm{CMen} B$ vaccine does not prevent carriage, ${ }^{6}$ so the objective of vaccination is to prevent cases. Public attention tends to focus on outbreaks among university students, who account for most cases of meningococcal B disease among young adults in the United States. However, cases also occur among the $62 \%$ of 18 -to24-year-olds in the United States who are not attending university. ${ }^{8}$

There are two general approaches to vaccine control of meningococcal B disease. In 2015, the U.K. government began a national infant vaccination program with $4 \mathrm{CMenB}$, with the aim of providing direct protection to one of the age groups at highest risk. ${ }^{9}$ The report by Ladhani et al., the results of which are also published in this issue of the Journal, highlights the effectiveness of that program, which is consistent with the experience in New Zealand. ${ }^{5,10}$ Others have focused attention on teenagers, the age group producing the highest numbers of meningococcal B cases. In the United States, limiting vaccination to students attending universities that have reported cases may seem logical. The risk in this group may be higher, but in fact all young adults are at risk. Therefore, the most logical approach to preventing meningococcal B disease among students is to provide a vaccine for all citizens in the very-high-risk age group of 17 to 18 years, which happens to be the usual age at which students enter university - and a time that for many students includes their first dating experience. In addition, students of all ages attending universities in which cases have occurred should be offered the vaccine and encouraged to accept it.

Disclosure forms provided by the author are available at NEJM.org.

From Murdoch Children's Research Institute and the University of Melbourne Department of Paediatrics, Melbourne, VIC, Australia; and the London School of Hygiene and Tropical Medicine, London. 
OPTION 2

Administer the Meningococcal B Vaccine Only to Students at Universities with an Outbreak

Kathryn M. Edwards, M.D.

The recommendation to administer meningococcal B vaccine only to students at universities where an outbreak has occurred is the best approach for several reasons. First, rates of meningococcal B disease are very low in the United States; the average annual incidence is only 0.17 cases per 100,000 population. Among the 1174 total cases of meningococcal disease reported during 2014-2016, only 166 occurred in persons 18 to 24 years of age, and only 88 of those cases were due to serogroup B. ${ }^{11}$ Thus, the costs of immunizing an entire birth cohort of 4 million people with two doses of meningococcal $B$ vaccine to prevent these few cases would be excessive. A recent study showed that the incremental cost per quality-adjusted life year gained with universal meningococcal $\mathrm{B}$ vaccination approached nearly \$14 million, as compared with the usual willingness-to-pay benchmark threshold of $\$ 150,000$ per quality-adjusted life year. ${ }^{12}$

The second reason that only targeted immunization during an outbreak is preferable is supported by the study by Marshall et al. published in this issue of the Journal. ${ }^{6}$ Investigators from Australia immunized more than 20,000 15-to18-year-old students with two doses of meningococcal $\mathrm{B}$ vaccines and showed that vaccination was not associated with a reduction in carriage - a finding that contrasts with the effect of the conjugate vaccines targeting serogroups $\mathrm{A}, \mathrm{C}, \mathrm{W}$, and $\mathrm{Y}$, which prevent colonization. Immunization with meningococcal $B$ vaccine does not prevent transmission and does not generate herd immunity.

Meningococcal B vaccines are directed against proteins expressed on meningococcal B strains, in contrast to the serogroup $\mathrm{A}, \mathrm{C}, \mathrm{W}$, and $\mathrm{Y}$ vaccines, which are directed against the polysaccharide capsules. Thus, not all infecting strains of serogroup B organisms share these proteins, and it is difficult to predict whether the vaccine strains will match the circulating strains. Data from one outbreak showed that more than 30\% of participants who were vaccinated with two doses of meningococcal B vaccine had low antibody titers, and therefore, there was no evidence of a protective response against the outbreak strain. ${ }^{13}$ The study conducted in England by Ladhani et al. shows that vaccine effectiveness for the prevention of invasive MenB disease in infants with a universal immunization program was only $60 \%$, with wide confidence intervals. ${ }^{10}$ Data on vaccine effectiveness from a large outbreak in Quebec, where more than $80 \%$ of nearly 60,000 older persons were immunized, showed similar rates. ${ }^{14}$ In addition, reports of vaccine failures in fully immunized adolescents during outbreaks supports the notion that the effectiveness of this vaccine falls short of that of other vaccines, such as measles or bacterial conjugate vaccines. ${ }^{15}$

Finally, rapid waning of vaccine-induced immunity has been clearly shown in serologic studies, ${ }^{16}$ a finding that has led to the recent recommendation by the CDC Advisory Committee on Immunization Practices that persons at high risk for meningococcal serogroup B disease should be given a booster dose of vaccine at 1 year after the primary series. Even if the entire birth cohort were immunized with meningococcal B vaccine, immunity for longer than a year could not be ensured.

Thus, for all the reasons outlined above, the best approach is to immunize only the students enrolled at the university with the outbreak. With robust use of the media to publicize the need for vaccination and with multiple participating clinics, the vaccine can be delivered to a large percentage of the university community.

Disclosure forms provided by the author are available at NEJM.org.

From the Division of Pediatric Infectious Diseases, Department of Pediatrics, Vanderbilt University Medical Center, Nashville.

This article was updated on February 20, 2020, at NEJM.org.

1. Soeters HM, McNamara LA, Blain AE, et al. University-based outbreaks of meningococcal disease caused by serogroup B, United States, 2013-2018. Emerg Infect Dis 2019;25:434-40.

2. Meningococcal B Immunisation Program. Adelaide: Department of Health and Wellbeing, Government of South Australia, 2019 (https://www.sahealth.sa.gov.au/wps/wcm/connect/public+ content/sa+health+internet/health+topics/health+conditions+ prevention+and+treatment/immunisation/immunisation+ programs/meningococcal+b+immunisation+program).

3. The routine immunisation schedule from autumn 2019. Public Health England, 2019 (https://assets.publishing.service .gov.uk/government/uploads/system/uploads/attachment_data/ 
file/824542/PHE_complete_immunisation_schedule_autumn _2019.pdf).

4. Villena R, Safadi MAP, Valenzuela MT, Torres JP, Finn A, O'Ryan M. Global epidemiology of serogroup B meningococcal disease and opportunities for prevention with novel recombinant protein vaccines. Hum Vaccin Immunother 2018;14:104257.

5. Arnold R, Galloway Y, McNicholas A, O'Hallahan J. Effectiveness of a vaccination programme for an epidemic of meningococcal B in New Zealand. Vaccine 2011;29:7100-6.

6. Marshall HS, McMillan M, Koehler AP, et al. Meningococcal $B$ vaccine and meningococcal carriage in adolescents in Australia. N Engl J Med 2020;382:318-27.

7. Olbrich KJ, Müller D, Schumacher S, Beck E, Meszaros K, Koerber F. Systematic review of invasive meningococcal disease: sequelae and quality of life impact on patients and their caregivers. Infect Dis Ther 2018;7:421-38.

8. Enhanced meningococcal disease surveillance report, 2017. Atlanta: Centers for Disease Control and Prevention, 2017 (https://www.cdc.gov/meningococcal/downloads/NCIRD-EMS -Report-2017.pdf).

9. JCVI position statement on use of Bexsero ${ }^{\circledR}$ meningococcal B vaccine in the UK. Public Health England, March 2014 (https:// assets.publishing.service.gov.uk/government/uploads/system/ uploads/attachment_data/file/294245/JCVI_Statement_on_MenB .pdf).

10. Ladhani SN, Andrews N, Parikh SR, et al. Vaccination of infants with meningococcal group B vaccine (4CMenB) in England. N Engl J Med 2020;382:309-17.

11. Mbaeyi SA, Joseph SJ, Blain A, Wang X, Hariri S, MacNeil JR. Meningococcal disease among college-aged young adults: 20142016. Pediatrics 2019;143(1):e20182130.

12. Leeds IL, Namasivayam V, Bamogo A, Sankhla P, Thayer WM. Cost effectiveness of meningococcal serogroup B vaccination in college-aged young adults. Am J Prev Med 2019;56:196204.

13. Basta NE, Mahmoud AAF, Wolfson J, et al. Immunogenicity of a meningococcal B vaccine during a university outbreak. $\mathrm{N}$ Engl J Med 2016;375:220-8.

14. De Wals P, Deceuninck G, Lefebvre B, et al. Impact of an immunization campaign to control an increased incidence of serogroup B meningococcal disease in one region of Quebec, Canada. Clin Infect Dis 2017;64:1263-7.

15. Mbaeyi S. Serogroup B meningococcal vaccine booster doses. Atlanta: Centers for Disease Control and Prevention, June 27, 2019 (https://www.cdc.gov/vaccines/acip/meetings/downloads/ slides-2019-06/Meningococcal-2-Mbaeyi-508.pdf).

16. Marshall HS, Richmond PC, Beeslaar J, et al. Meningococcal serogroup B-specific responses after vaccination with bivalent rLP2086: 4 year follow-up of a randomised, single-blind, placebocontrolled, phase 2 trial. Lancet Infect Dis 2017;17:58-67.

DOI: 10.1056/NEJMclde1916746

Copyright (c) 2020 Massachusetts Medical Society. 